\title{
Numerical studies of wrinkling phenomenon in inflatable hyperelastic membranes undergoing multiaxial loadings.
}

\author{
H. DAANOUNI, Y. TILLIER, F. BAY \\ Mines ParisTech, CEMEF - Centre de Mise en Forme des Matériaux, CNRS UMR 7635, BP 207, \\ 1 rue Claude Daunesse, 06904 Sophia Antipolis Cedex, France \\ Email: Hicham.daanouni@mines-paristech.fr; Yannick.tillier@mines-paristech.fr; Francois.Bay@mines-paristech.fr;
}

\begin{abstract}
Thin membranes subject to multiaxial loadings may exhibit wrinkling phenomena. Modelling this phenomenon can be interesting if one wants to understand the origin of wrinkling and optimise the design of the membrane in order to avoid such kind of defect. The work, presented here aims at predicting wrinkling phenomena in thin hyperelastic membranes undergoing large deformation due to multiaxial loadings -using a finite element modelling tool for 3-D configurations. The constitutive equations used for modelling the membrane behaviour are hyperelastic models (the neo-Hookean model, the Mooney-Rivlin one and a third one with a higher degree of non-linearity). They have been implemented in a finite element code developed in our laboratory FORGE2005®, which is based on a mixed pressure-velocity formulation. The software is then applied to the deformation of a hyperelastic spherical form undergoing internal pressure. We show that the ability of tetrahedral elements to correctly predict wrinkling phenomena strongly depends on the various numerical parameters used (load increment, pressure ratio, mesh size, number of elements ...)
\end{abstract}

KEYWORDS: Hyerelastic materials, thin membrane, buckling, wrinkling, large deformation

\section{INTRODUCTION}

Modelling of wrinkling in thin membranes undergoing multiaxial loadings can be interesting in the design stage. Up to this date, this problem has been mainly treated as a plane stress problem and the work that has been done has mainly focused on determining the wrinkling regions [1]. These approaches do not enable to access the shapes of the wrinkles - i.e amplitude and wavelength. The wrinkling of hyperelastic membranes has been investigated by many authors. Among those approaches, two kinds of finite elements are commonly used: shell elements which have zero bending stiffness and volumic elements. Our approach clearly belongs to the second category which consists in discretizing the membrane with tetrahedron $\mathrm{P} 1+/ \mathrm{P} 1$ elements [2], the constitutive equation used is an hyperelastic model which is developed on Forge 2005® (Neo-hookeen, MooneyRivlin and Fung [3]).

Objective of this work is to study the ability of tetrahedral elements to model the wrinkling phenomena. To this end, we investigate two cases: a sphere undergoing both internal pressure and indentation (Figure 1a) and the buckling of an airbag (Figure 1b).

We shall first present a brief review of hyperelasticity constitutive laws. We shall then introduce the hyperelastic models selected for modeling the membrane and the numerical developments that we have done for introducing these models in Forge 2005®. Finally, and after validation of our new software, we present a sensitivity study of wrinkles with respect to physical and numerical parameters.

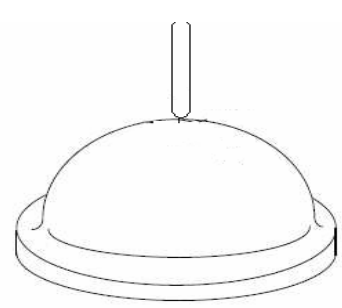

(a)

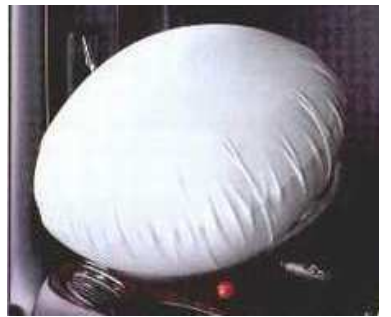

(b)
Figure 1: cases studied.

\section{MECANICHAL FORMULATION}

In this section, we present a brief summary of the hyperelastic materials and their main scientific basis. We then present hyperelastic models implemented in FORGE 2005®.

\subsection{HYPERELASTICITY}

A hyperelastic material has a linear or non-linear behaviour with respect to strain, path-independent, and recovers completely from large deformation. In our case, we assumed the material to be isotropic and nearly or purely incompressible.

The stress can be expressed as an energy density function (W) that verifies: 


$$
S=2 \frac{\partial W}{\partial C}=2 \sum_{I=1}^{2} \frac{\partial w}{\partial I_{i}} \frac{\partial I_{i}}{\partial C}
$$

Where $\mathrm{S}$ denotes the second Piola-Kirchhoff tensor, $\mathrm{C}$ the right Cauchy-Green strain tensor $C=F^{T} F$, $F=\operatorname{Grad} x$ the deformation gradient. Note that the energy function is expressed as a function of the invariants of $\mathrm{C}, W=W\left(I_{1}, I_{2}\right)$. The invariants of the right Cauchy-Green tensor are given by $I_{1}=\operatorname{tr}(C)$, $I_{2}=\frac{1}{2}\left[(\operatorname{tr}(c))^{2}-\operatorname{tr}\left(C^{2}\right)\right] \quad$ and $\quad I_{3}=\operatorname{det}(C)=1$ (incompressibility hypothesis).

The Cauchy stress tensor $\sigma$ is related to the second PiolaKirchhoff $S$ tensor by:

$$
S=J F^{-1} \sigma^{T}\left(F^{-1}\right)
$$

Where $J=\operatorname{det} F$.

From (1) and (2) we can easily find the Cauchy stress tensor expression:

$$
\sigma=2\left[\frac{\partial W}{\partial I_{1}} B-\frac{\partial W}{\partial I_{2}} B^{-1}\right]-P^{\prime} I=\sigma^{\prime}-P^{\prime} I
$$

$\sigma^{\prime}$ is not the deviator stress tensor.

\subsection{MODELS IMPLEMENTED}

Several models are proposed in literature. A recent study on hyperelastic models of VERRON et al [4] has reviewed twenty different hyperelastic constitutive models for rubber, from the Mooney-Rivlin (1940) to the micro-sphere model (2004) in the incompressible case. For our study we have selected three models, the neoHookean model, the Mooney-Rivlin one and a third one with a higher degree of non-linearity - the Fung model [3].

The constitutive laws of these three models are listed in the table below.

Table 1: Constitutive equations

\begin{tabular}{ccc}
\hline Model & Energy function $W$ & $\sigma^{\prime}$ \\
\hline $\begin{array}{c}\text { Neo- } \\
\text { Hookean }\end{array}$ & $W=c_{1}\left(I_{1}-3\right)$ & $\sigma^{\prime}=2 c B$ \\
\hline $\begin{array}{c}\text { Mooney- } \\
\text { Rvilin }\end{array}$ & $W=c_{1}\left(I_{1}-3\right)+c_{2}\left(I_{2}-3\right)$ & $\sigma^{\prime}=2 c_{1} B-2 c_{2} B^{-1}$ \\
\hline Fung & $W=\frac{\mu}{2 \gamma}\left(e^{\gamma\left(I_{1}-3\right)}-1\right)$ & $\sigma^{\prime}=\mu\left(e^{\gamma\left(l_{1}-3\right)}\right) B$ \\
\hline
\end{tabular}

\section{NUMERICAL RESOLUTION}

In this section, we present a finite element approach for solving problem (4). The total or nearly total incompressibility constraint has led us to use a mixed formulation in term of velocity and pressure fields [5]. A time and a space discretization are used.

\subsection{WEAK MIXED FORMULATION}

In the case of a mixed formulation, for an incompressible hyperelastic material, the solution of the problem must satisfy the following system for any virtual (velocity, pressure) $\left(v^{*}, p^{*}\right)$ couple:

$$
\left\{\begin{array}{l}
\int_{\Omega} s^{t+\Delta t}(v): \varepsilon\left(v^{*}\right)-\int_{\partial \Omega_{e}} T^{t+\Delta t}(v) \cdot v^{*}-\int_{\Omega} p^{t+\Delta t} d i v\left(v^{*}\right)-\int_{\Omega} \rho g \cdot v^{*}=0 \\
\int_{\Omega} p^{*}\left(\operatorname{div}\left(v^{*}\right)+\frac{\operatorname{det} F^{t}-1}{\operatorname{det} F^{t} \Delta t}\right)=0
\end{array}\right.
$$

System (4) is an incremental mixed formulation of the problem.

\subsection{FINITE ELEMENT DISCRETIZATION}

\subsubsection{Time discretisation}

The unknown of the problem (4) is the couple (displacement, pressure). For a time step $\Delta t$ small enough, we can assume that we have a constant velocity current the time step $\Delta t$. We update the displacement field using an explicit integration scheme:

$$
u^{t+\Delta t}=u^{t}+\Delta t . \mathrm{V}
$$

\subsubsection{Spatial discretization}

In forge $2005 \AA$ we use a linear tetrahedral elements, which have a bubble function for velocity interpolation. These elements are represented on the figure (2). More details on this type of elements can be found in [2].

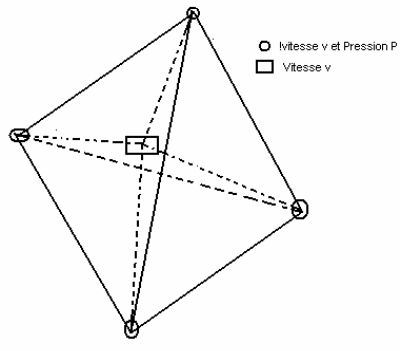

Figure 2: interpolation in mini-elements.

The velocity $v_{h}$ and pressure $p_{h}$ fields discreet are given by:

$$
\begin{aligned}
& v_{h}=v_{h}^{l}+v_{h}^{b}=\sum_{k=1}^{n b n o e} V_{k} N_{k}^{l}+\sum_{i=1}^{n \text { nbelt }} V_{i} N_{i}^{b} \\
& p_{h}=\sum_{k=1}^{\text {nbnoe }} P_{k} N_{k}^{l}
\end{aligned}
$$

$N^{l}, N^{b}$ are respectively linear and bubble functions.

\section{VALIDATION \& APPLICATIONS}

The aim of this section is to validate the implementation presented before, and prove the ability of Forge $2005 \AA$ 
to model the wrinkling phenomena introduced previously.

\subsection{VALIDATION}

In order to test the validity of the code, we use a uniaxial tension test of a cube quite popular in literature $[6,7]$. The cube of $5 \mathrm{~mm}$ length (figure $3 \mathrm{a}$ ) is stretched over 20 $\mathrm{mm}$. One advantage of this case is that we can easily determine the analytical solution. For example the analytical solution of the Mooney-Rivlin model is given by:

$$
\sigma_{z z}=2 C_{1}\left[\frac{L^{2}}{L_{0}^{2}}-\frac{L_{0}}{L}\right]-2 C_{2}\left[\frac{L_{0}^{2}}{L^{2}}-\frac{L}{L_{0}}\right]
$$

Where $C_{1}$ and $C_{2}$ denote the material coefficients, $L_{0}$ the initial length.

The following figure displays the results of a simulation with Forge $2005 \AA$. The constitutive equation considered is a Mooney-Rivlin one. A bilateral sticking contact is used between the cube and the upper and lower dies.

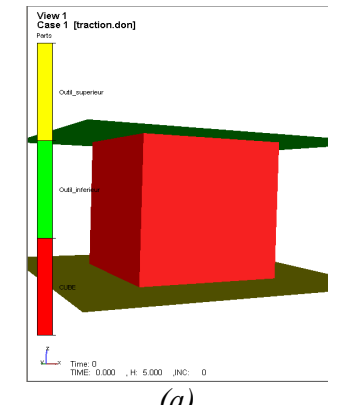

(a) (a)

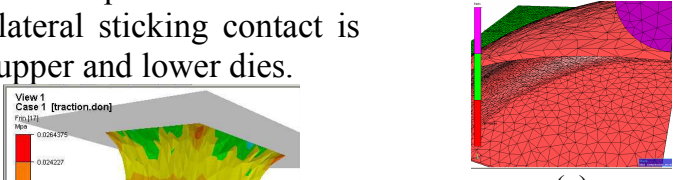

an indentation on the top (figure 1a). Case 2 displays a round airbag (figure 1b), subject only to an internal pressure. For both cases, the thickness of the membrane used is $1 \mathrm{~mm}$. We focus here on the selection of numerical parameters best suited to observe wrinkling using tetrahedral elements.

\subsubsection{Mesh size influence}

First, the mesh size has been studied - in particular the number of elements in the thickness of the structure and its influence on buckling in the membrane.

The constitutive equation chosen for this case is a neoHookean model, with $C_{1}=0.0033 \mathrm{MPa}$. The deltapressure imposed is $\triangle P=10^{-4} M P a$ and maintained constant. The die displacement is $10 \mathrm{~mm}$ in (-y) direction.

In figure 5, we present three types of meshing, with one element (figure 5a), two elements (figure 5b) and three elements (figure 5c) in the thickness.
Figure 5: different meshes tested

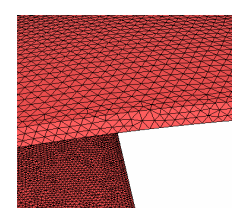

(c)
For reducing calculation time we use two symmetry planes.

The results clearly show that, when only one element is used in the thickness, we get no wrinkling (figure 6a). When we use two elements in the thickness we obtain a pseudo apparition of the phenomenon (figure 6b). When using three elements (or more) we clearly observe wrinkling on the top of the membrane as we can see on figure $6 \mathrm{c}$.

This result was compared to the analytical solution. Both solutions, as we can see on the figure (4), are very close.

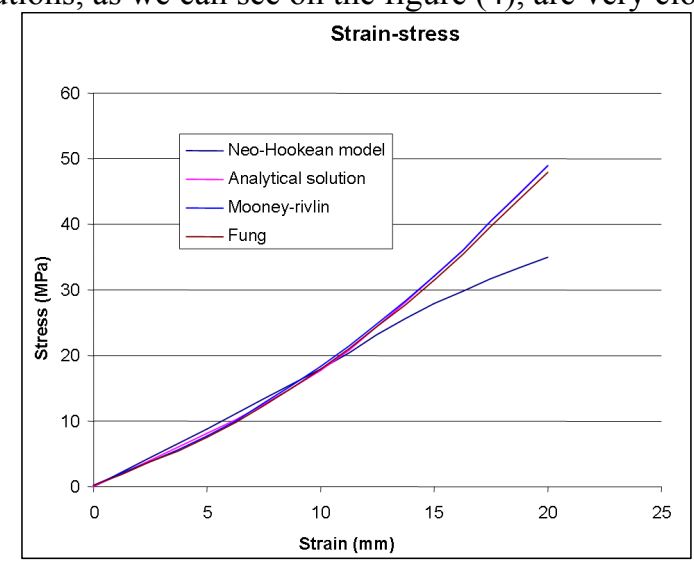

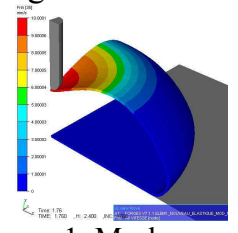

1. Mesh a

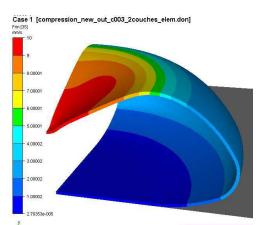

2. Mesh $\bar{b}$

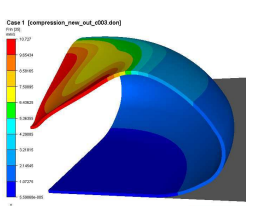

3. Mesh c
Figure 6: velocity field

\subsubsection{Pressure ratio (internal vs. external)}

Another study was conducted on the influence of the internal pressure. Several ratios between the internal and the external pressures have been tested. The main results are summarized in the following table.
Figure 4: Comparison between Forge $2005 \AA$ results and analytical solution for the tension test

\subsection{INFLATABLE STRUCTURES APPLICATION}

Two examples are presented here. Case 1 deals with a spherical form undergoing both an internal pressure and 
Table 2: Pressure ratios tested

\begin{tabular}{|c|c|c|c|}
\hline Case & 1. & 2. & 3. \\
\hline$|\Delta \mathrm{P}|(\mathrm{MPa})$ & $10^{-4}$ & $10^{-3}$ & $10^{-2}$ \\
\hline
\end{tabular}

Results are presented in figure 7.

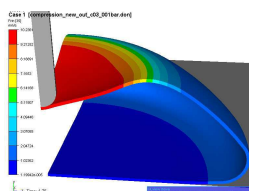

(a) (b)

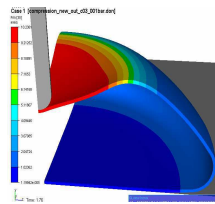

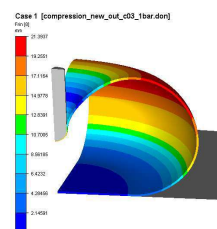

(c)
Figure 7: Velocity field (Forge 2005®)

We observe no wrinkles when pressure is low (figure 7a) or strong (figure 7c); for medium pressure we clearly observe wrinkles (figure $7 \mathrm{~b}$ ). The difference between internal and external pressure is a major factor for modelling the wrinkling phenomena.

After full 3-D reconstruction by symmetry, we obtain:

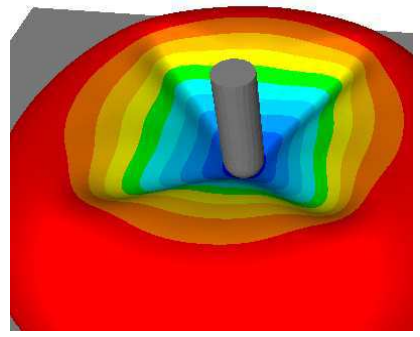

Figure 8: Results after reconstruction (Forge 2005®) displacements isovalues

\subsubsection{Case 2: inflatable structure; round airbag}

The second case studied is a membrane undergoing only an internal pressure. Here we consider a round airbag. We keep all the parameters introduced before (time step, number of elements in the thickness).

The internal pressure is constant and equal to $4.410^{-5}$ $\mathrm{MPa}$. A bilateral sticking contact in the centre of the lower face of the airbag is considered. The neo-Hookean model is used for the membrane material. The membrane is $1 \mathrm{~mm}$ thick. We have three elements through the thickness. Buckling appears clearly in the equatorial part of the airbag in Figure 9.
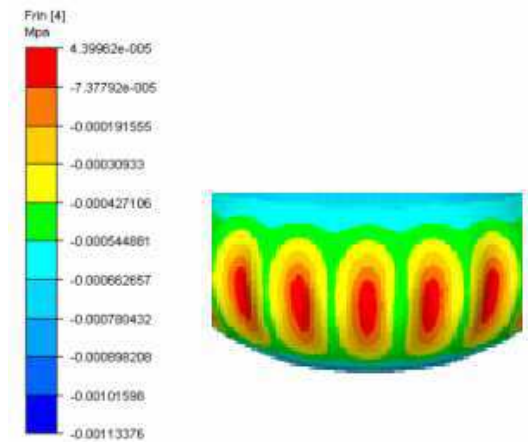

Figure 9: Pressure field (Forge 2005®)

\section{CONCLUSIONS}

The aim of this work has been to develop a reliable software and define numerical and physical parameters enabling to correctly predict wrinkling phenomena in thin membrane undergoing multiaxial loading in large deformation. Numerical examples selected have shown the ability of tetrahedral element to model wrinkling and buckling in thin membrane. These results are strongly dependent on the various numerical parameters used as time step, pressure ratio, mesh size, number of elements. Computation time remains a problem, but this method provides more information through the thickness than other methods.

We plan in the future to model wrinkling phenomena in thin membranes containing a deformable body (or several deformable bodies). The contact algorithm, as well as the mesh quality will be analysed. Tetrahedral elements will be used for the internal bodies too.

\section{REFERENCES}

[1] Diaby A, Le Van A, Wielgosz C. "Buckling and wrinkling of prestressed membranes". Science direct. Finite Elements in Analysis and Design 42 (2006) 992 - 1001.

[2] Coupez T., "Stable stabilized finite element for 3D forming calculation". CEMEF. Rapport interne (1996).

[3] FungY.C. "Elasticity of soft tissues in simple elongation" American Journal of physiology, 213, pp. 1532-1544.

[4] Marckmann G., Verron E., "Comparison of hyperelastic models for rubberlike materials", Rubber Chemistry and Technology, 79(5), 835-858, 2006.

[5] Chenot J-L, Massoni E. "Finite element modelling and control of new metal forming processes" CEMEF; 2006

[6] Agassant J.-F., Avenas P., Sergent J.-P., Vergnes B., Vincent M., "La mise en forme des matières plastiques ". Technique \& Documentation. ISBN : 2-7430-0016-3. Editions Lavoisier (1996).

[7] Tillier Y. et al., "Finite element modeling for soft tissue surgery based on linear and nonlinear elasticity behaviour", Computer Aided Surgery, Volume 11, 2006(2),63 - 68 